\title{
Tecnologia educacional voltada à avaliação de feridas em uma Unidade de Terapia Intensiva
}

\author{
Educational technology aimed at the evaluation of \\ wounds in an Intensive Care Unit
}

\author{
Ana Zélia Silva Fernandes de Sousa', Joana Carla da Silva Souza', \\ Daniele Lima dos Anjos Reis', Kátia Simone Kietzer² \\ Universidade do Estado do Pará, Tucuruí (PA), Brasil. \\ ${ }^{2}$ Universidade do Estado do Pará, Belém (PA), Brasil.
}

\begin{abstract}
RESUMO
Introdução: A Unidade de Terapia Intensiva (UTI) é um setor crítico do hospital destinado aos pacientes que necessitam de vigilância contínua por estado instável, e conseguinte a isso passível a apresentar outras alterações comuns, como feridas ou lesões de pele. Neste contexto o enfermeiro deve estar capacitado para realizar uma avaliação efetiva dessas feridas, tornando-se adequado e vantajoso a adoção de uma tecnologia em saúde para auxiliá-lo. Neste sentido, o objetivo desse estudo é relata as experiências e a ação educativa realizada na apresentação da ficha de avaliação de feridas, para a equipe de saúde do setor da UTI adulto do HRT. Desenvolvimento: Trata-se de um relato de experiência dos autores durante estágio supervisionado. A problematização foi escolhida como metodologia e utilizou-se roda de conversa com a equipe para realização de uma ação educativa, que culminou na apresentação da ficha de avaliação de feridas e explanação dos itens contidos nela. Evidenciou-se um feedback positivo, pois os profissionais mostraram-se satisfeitos e assertivos em aceitar a aplicação da escala de avaliação de risco de lesão por pressão no ambiente assistencial, assim, constata-se que uma atividade de Educação Permanente, pautada nas reais necessidades da população-alvo, pode provocar mudanças significativas na promoção da saúde. Considerações finais: É de essencial importância a contribuição desta tecnologia para o engajamento do planejamento do cuidado ao paciente com a mobilidade comprometida, em grande risco de lesão por pressão, o que colabora na execução de ações rápidas e competentes para a prevenção, direcionando as intervenções de enfermagem, garantindo uma avaliação clínica minuciosa, além de gerar benefícios ao profissional, ao serviço e especialmente aos pacientes.
\end{abstract}

Recebido: Maio 21, 2018 Aceito: Mar. 29, 2019

\section{COMO CITAR ESTE ARTIGO}

Sousa AZSF, Souza JCS, Rei DLA, Kietzer KS. Tecnologia educacional voltada à avaliação de feridas em uma Unidade de Terapia Intensiva. Interdisciplinary Journal of Health Education 2019 Jan-Dez:4(1-2):55-61 https://doi.org/10.4322/ijhe.2018.015

\section{CORRESPONDÊNCIA}

Ana Zélia Silva Fernandes de Sousa Universidade do Estado do Pará Rua Quatro, 20, Santa Mônica, CEP 68455-210, Tucuruí (PA), Brasil anazeliafernandes19@gmail.com

\section{FONTE DE FINANCIAMENTO}

Nenhuma.

\section{CONFLITO DE INTERESSE}

Os autores declararam não

haver conflitos de interesse.

O estudo foi realizado no Núcleo de Tucuruí-PA, Hospital Regional de Tucuruí, Universidade do Estado do Pará, Tucuruí (PA), Brasil.

Todos os autores leram e aprovam a versão final submetida ao Interdisciplinary Journal of Health Education (IJHE).
PALAVRAS-ChAVE: Tecnologia Educacional. Lesão por pressão. Cuidados críticos.

\begin{abstract}
Introduction: The Intensive Care Unit (ICU) is a critical area of the hospital for patients who need continuous surveillance because of an unstable state, and consequently may present other common alterations, such as injuries or skin lesions. In this context, the nurse should be able to perform an effective evaluation of these wounds, making it appropriate and advantageous to adopt the health technology to assist it. The research aimed to contribute to nurses and ICU sector team by presenting the wounds assessment sheet in order to promote health and also highlight the importance of care. Development: This is an experience report of the authors during the supervised stage. The problem was chosen as a methodology and a conversation with the team was used to carry out an educational action, which culminated in the presentation of the assessment sheet of wounds and explanation of the items contained in it. A positive feedback was evidenced, since the professionals were satisfied and assertive in accepting the application of the scale of evaluation of risk of injury by pressure in the care environment, thus, it is verified that a Permanent Education activity, based on the real needs of the target population, can cause significant changes in health promotion. Final considerations: The contribution of this technology to the engagement of patient care planning with compromised mobility, at great risk of pressure injury, is essential, which contributes to the execution of fast and competent actions for prevention, directing interventions of nursing, guaranteeing a thorough clinical evaluation, besides generating benefits to the professional, the service and especially the patients.
\end{abstract}

KEYWORDS: Educational Technology. Pressure injury. Critical care. 


\section{Contextualização}

A Unidade de Terapia Intensiva (UTI) é um setor crítico do hospital destinado aos pacientes que necessitam de vigilância contínua e suporte terapêutico especializado, pois as condições clínicas alternam-se entre limites estreitos de normalidade e anormalidade. Devido a essa criticidade e o tipo de assistência prestada, outras alterações também podem estar presentes, como por exemplo, feridas ou lesões de pele $^{1,2}$.

As feridas são alterações dermatológicas que quando não tratadas adequadamente são responsáveis por lesões maiores e/ou perda de função da região acometida. Representam um grande problema de saúde que envolve diversos fatores relacionados ao paciente e ao seu meio externo ${ }^{3}$. E a ascensão desse problema em clientes hospitalizados, especialmente em UTI, é um parâmetro negativo para a qualidade assistencial, já que a sua gênese se associa diretamente ao aumento do tempo de hospitalização, do risco de complicações e dos custos ${ }^{4}$.

Neste contexto todos os profissionais que prestam cuidado ao paciente devem estar capacitados para realizar uma avaliação efetiva das feridas, devendo levar em consideração diversos fatores como causa e tempo de existência da ferida e presença ou não de infecção. Além disso, é preciso avaliar a dor, a presença de edema, a extensão e a profundidade da ferida e as características do leito da ferida, da pele perilesional e do exsudato. É necessário ainda, realizar um diagnóstico preciso, identificar o período evolutivo cicatricial e tratar a ferida de forma adequada, bem como aproveitar ao máximo o desempenho das equipes de saúde e dos materiais disponíveis ${ }^{5}$.

O enfermeiro atuante diretamente no cuidado ao paciente crítico com lesões de pele assume uma conduta de vital importância, sendo responsável por uma avaliação clínica diária minuciosa aos pacientes internados na UTI, considerando o nível de gravidade e instabilidade clínica desses pacientes. No que se refere à avaliação de feridas e curativos, o cuidado do enfermeiro é de suma importância, sendo necessária a atualização contínua deste profissional quanto ao seu conhecimento e as práticas baseadas em estudos científicos, complementando um cuidado diário mais efetivo pela equipe de enfermagem ${ }^{6}$.

O termo tecnologia é um conjunto de ações que incluem métodos, procedimentos, técnicas, equipamentos e outros instrumentos aplicados ao conhecimento científico, englobando saberes diversos ${ }^{7}$. Em síntese, as tecnologias não são um fim e, sim, um meio para o profissional enfermeiro prestar um cuidado humanizado, que garanta melhoria da qualidade de vida do sujeito ${ }^{8}$.

Desse modo, tecnologia de cuidado são recursos tanto humanos como materiais, onde direta ou indiretamente, estão a serviço do cuidado. Como desafio, a enfermagem deve provocar a inserção da tecnologia na prática em saúde, abrangendo as demandas sociais e refletindo quanto as questões éticas que permeiam a utilização das tecnologias frente a assistência9.

Neste contexto, os enfermeiros necessitam empenhar-se na busca por inovações relacionadas a sua prática, sendo inúmeras as formas de utilização e aproveitamento dos aparatos tecnológicos, a permanente atualização beneficia tanto o profissional quanto o cliente, contribuindo desta forma na prática do cuidar.

Após a vivência no estágio supervisionado em UTI, foi constatado que os pacientes admitidos na unidade não recebiam uma avaliação sobre os riscos que contribuem para o desenvolvimento de lesões na pele, e os que já apresentavam alguma ferida não recebiam avaliação diária para acompanhar o processo de cicatrização. Constatou-se, então, a necessidade de desenvolver uma tecnologia de cuidado voltada para a avaliação de feridas em pacientes hospitalizados admitidos na UTI, haja vista que a identificação de riscos que contribuem para o desenvolvimento de lesões na pele e acompanhamento do processo de cicatrização dos pacientes internados depende de 
avaliações sistematizadas e cuidados de acordo com cada momento da evolução do processo de cicatrização.

Neste sentido, o objetivo desse estudo foi relatar a experiência de elaborar uma "Ficha de Avaliação de Feridas" para pacientes internados na UTI adulto do Hospital Regional de Tucuruí (HRT), bem como de executar uma ação educativa com a equipe de saúde do referido setor relacionado ao tema feridas e curativos e apresentação da ficha elaborada.

\section{Descrição da experiência}

Trata-se de um relato de experiência de uma atividade realizada pelos autores durante o "Estágio Supervisionado em UTI adulto", componente curricular obrigatório do $5^{\circ}$ ano do Curso de Graduação em Enfermagem da Universidade do Estado do Pará (UEPA), no HRT, unidade hospitalar pública de média e alta complexidade. A realização dessa atividade ocorreu entre o período de 8 a 24 de maio de 2017, após a prévia autorização da Direção, do Núcleo de Educação Continuada e Coordenação da UTI adulto do HRT.

O relato de experiência apresenta uma reflexão sobre uma ação ou um conjunto de ações que acercam situações vivenciadas no âmbito profissional de interesse da comunidade científica, sendo uma ferramenta da pesquisa descritiva ${ }^{10}$.

O projeto desta pesquisa não necessitou passar pelo Comitê de Ética em Pesquisa pelo fato de se tratar de um relato de experiência dos autores, com o consentimento do local onde ocorreu o estágio curricular obrigatório, utilizando como forma de coleta e dados: a observação estruturada (pesquisador participante), participação nas atividades (ação educativa) e diário de campo, mantendo sempre o sigilo de informações.

A exemplo de uma metodologia nova, onde Paulo Freire enfatiza a autonomia do discente tornando-o um ser crítico e reflexivo, nasce a Problematização que possui como eixo básico a ação-reflexão-ação, que tem sua origem a partir do momento que o docente permite o aluno a se perceber como ser inserido no mundo e desta forma responder os novos desafios. Os problemas que serão estudados devem partir de um cenário real tendo seu trabalho político pedagógico marcado por uma postura crítica da educação ${ }^{11}$.

A problematização foi escolhida como metodologia para o planejamento das atividades propostas, sendo descrita pelo Arco de Maguerez (Figura 1), composto por cinco etapas: observação do problema, identificação dos fatores mais importantes acerca do problema, estudo das causas do problema, desenvolvimento de hipóteses de solução e, por fim, ações para resolver o problema ${ }^{12,13}$.

Durante a fase de Observação da realidade, os discentes foram instigados pela proposta das Atividades Integrativas em Saúde (AIS) lançada pela Universidade, que possuía como tema gerador a criação de uma "Tecnologia do Cuidado" voltada ao campo de estágio atuante.

Desta forma foi observado que os pacientes admitidos na UTI adulto do hospital em questão na sua maioria encontravam-se acamados, em estado de consciência comatoso ou sedado, tendo, portanto, dificuldades ou impossibilidade de locomoção e mobilidade no leito, fatores estes contribuintes para formação das lesões por pressão.

Observou-se também que tais pacientes não eram avaliados pela equipe de enfermagem de maneira sistemática, por meio de algum instrumento formal, quanto aos riscos somatórios para o desenvolvimento de uma lesão e nem quanto à evolução do processo de cicatrização das lesões pré-existentes, de forma que as informações referentes a estas questões eram muitas vezes inexistentes ou encontradas na folha de evolução no prontuário do paciente de maneira imprecisa e incompleta, sem possibilitar a elaboração e execução de um plano de cuidados de enfermagem personalizado e eficaz. 
Por isso, a Tecnologia de Cuidado escolhida para a ação educativa foi criação de uma "Ficha de Avaliação de Feridas", como instrumento norteador das atividades do Enfermeiro da UTI adulto do referido hospital. Os pontos-chaves foram traçados por meio de uma análise reflexiva, pautados nos princípios científicos relacionados à etiologia, formação, caracterização, cicatrização, curativos e avaliação sistemática de feridas, bem como na importância da implantação desta ferramenta para o trabalho dos enfermeiros intensivistas.

Em seguida, partiu-se para a teorização, onde buscou-se na literatura científica e bases de dados da área da saúde, documentos para o estabelecimento de um suporte teórico-cientifico e atualização das informações de forma que nos guiassem no planejamento da ação educativa e na elaboração da tecnologia de cuidado.

A hipótese de solução gerada, foi a de elaborar uma ficha Tabela 1 para avaliação das lesões já existentes em alguns pacientes; de analisar os riscos de pacientes sem lesões desenvolvê-las e de sintetizar, agilizar e padronizar o trabalho da equipe profissional em relação à avaliação desse paciente; contribuindo, dessa forma, para a organização e melhoria da qualidade da assistência de enfermagem prestada.

O ápice desta atividade, ou seja, a aplicação à realidade culminou na realização de uma ação educativa, que ocorreu no dia 24 de maio de 2017, no período da manhã, em um espaço reservado no próprio setor da UTI. Participaram todos os técnicos de

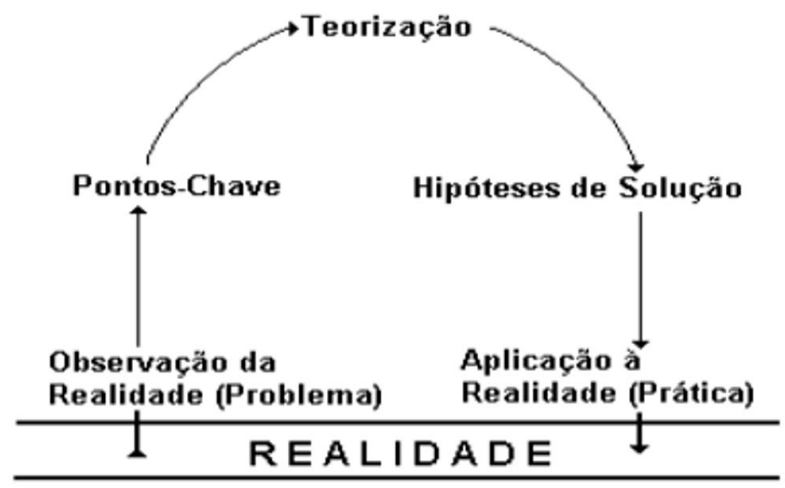

Figura 1. Método do Arco de Maguerez.

Tabela 1. Estruturação da ficha de avaliação de feridas.

\begin{tabular}{ll}
\multicolumn{1}{c}{ TÓPICO } & \multicolumn{1}{c}{ CONTEÚDo } \\
\hline Informações do Paciente & Descrição do nome, dias de internação, situação quanto a mobilidade. \\
\hline Antecedentes Pessoais & Investigação quanto a patologias pré-existentes e estilo de vida. \\
\hline Fatores Contribuintes & $\begin{array}{l}\text { Investigação sobre a realização de algum tratamento específico ou } \\
\text { condição favorável. }\end{array}$ \\
\hline $\begin{array}{l}\text { Avaliação de Risco para } \\
\text { desenvolvimento da lesão }\end{array}$ & Utilização da escala de Braden para verificar o score de risco. \\
\hline Etiologia da Lesão & $\begin{array}{l}\text { Investigação quanto a pré-existência de condições para desenvolvimento } \\
\text { da lesão. }\end{array}$ \\
\hline Tempo da Ferida & Descrição em dias a duração da lesão. \\
\hline Localização & Identificação dos pontos afetados pelas lesões. \\
\hline Tamanho & $\begin{array}{l}\text { Descrição em centímetro do comprimento, largura e profundidade da } \\
\text { lesão. }\end{array}$ \\
\hline Leito da Ferida & Descrição de qual/quais tecido (s) está (ao) presente (s). \\
\hline Bordas da Ferida & Caracterização do tecido presente. \\
\hline Caracterização da Ferida & Descrição quanto a profundidade e presença de sinais flogísticos. \\
\hline Estágio da Lesão & Classificação quanto a evolução da lesão. \\
\hline Exsudato & Se presente o exsudato, descrever suas características. \\
\hline Dor da Ferida & Avaliação feita com escala de faces de dor. \\
\hline Curativos & Descrição do tipo de curativo. \\
\hline Observações & Espaço para descrição de anotações necessárias. \\
\hline
\end{tabular}


enfermagem, a enfermeira e a fisioterapeuta do setor, totalizando 07 participantes. A ação iniciou-se com a apresentação das discentes e da docente, seguindo de uma breve explanação sobre os conceitos básicos acerca do assunto "feridas e curativos", apresentação do objetivo da tecnologia elaborada e explanação dos itens contidos nela.

Por meio da roda de conversa executada, os participantes puderam realizar questionamentos, sanar dúvidas geradas durante o momento e sugerir alterações para a melhoria da qualidade do impresso. Notou-se que durante toda a ação ocorreu envolvimento e contribuição dos participantes, e os mesmos mostraram-se satisfeitos e interessados quanto ao tema apresentado, já que se tratava de uma necessidade explícita à melhoria do trabalho realizado pela equipe de enfermagem do setor em relação ao cuidado com feridas, especialmente lesões por pressão.

Para a realização da ação educativa adveio algumas dificuldades para sua implementação efetiva, dentre elas, a mais relevante, fora conseguir reunir toda a equipe profissional da unidade de terapia intensiva para a explicitação da tecnologia, os quais resultou um total de sete participantes na referida ação, constituindo-se um momento rico, no qual se estabeleceu o movimento das relações, ou seja, a escuta, o vínculo, o perpassar do conhecimento cientifico e a educação permanente.

Desta forma, levando em consideração a relevância da tecnologia de cuidado elaborada e a aceitação por parte da equipe da UTI adulto do HRT, a "Ficha de Avaliação de Feridas" foi encaminhada para análise e aprovação da Gerência de Enfermagem e Direção Técnica, com o apoio do Núcleo de Educação Continuada, para posterior aplicação de possíveis correções, validação da tecnologia de cuidado e implantação no setor de UTI adulto como impresso padronizado.

\section{Resultados e impactos}

No ambiente da UTI, rotineiramente ocorrem danos desnecessários, conhecidos como eventos adversos, que acabam por acarretar aos pacientes lesões medíveis, maior tempo de internação ou óbito. A qualidade da assistência de enfermagem prestada ao paciente em estado crítico se torna um desafio para equipe, a fim de evitar danos. Para tanto, é necessário proporcionar estratégias que possam envolver a instituição e a equipe multidisciplinar atuante na $\mathrm{UTI}^{14,15}$.

As tecnologias do cuidado têm por objetivo expandir a aplicabilidade da atividade humana nas mais variadas esferas ${ }^{7}$, desta forma a elaboração da ficha de avaliação de feridas teve o objetivo de buscar minimizar a exposição a danos desnecessários e proporcionar o aumento da eficácia da equipe ao cuidar do paciente no ambiente da UTI em especial aqueles que apresentam feridas e necessitam de cuidado com curativos.

A educação é vista como o processo dinâmico de ensino-aprendizagem, a qual deve ser ativa e permanente, lapidando as pessoas e as preparando para se tornarem melhores na execução do seu papel na sociedade, promovendo, portanto, a transformação e libertação do homem ${ }^{16,17}$.

A realização da roda de conversa juntamente a equipe multidisciplinar da UTI, proporcionou um espaço para troca de saberes, a fim de proporcionar aos profissionais uma tecnologia que lhes garantissem um olhar mais amplo ao paciente e desta forma contribuir para evitar ou reduzir danos principalmente aos portadores de algum fator de risco para desenvolver lesões na pele.

No estudo de Costa et al. ${ }^{18}$ utilizou-se a escala de Braden, no Centro de Terapia Intensiva e foi possível evidenciar uma grande sensibilidade e especificidade, para a predição do grau de risco do paciente para o desenvolvimento de Lesão por Pressão, o que impactou na identificação dos fatores individuais de risco e condições predisponentes influenciadoras. Ainda, fomenta a importância da utilização das 
escalas como tecnologia do cuidado, e considera imprescindível o aprofundamento em investigações futuras, o que corrobora com o presente estudo.

Mediante a isso, é de locupletar-se a preocupação que há em contribuir para a produção do conhecimento contínuo dos profissionais, especialmente a enfermagem por estar presente diretamente na assistência a pacientes com feridas, e lesões por pressão, bem como alertar a equipe quanto a utilização de métodos assistenciais, tais como, as escalas de risco abordadas e apresentadas nesta linha de pesquisa. Nesse sentido, considera-se, também, como fundamental, contribuir para o fortalecimento tecnológico do processo de cuidar em enfermagem.

Nessa experiência, os profissionais apresentaram-se receptivos, interessados e participativos com as atividades executadas. Além desse feedback proporcionado no dia da atividade e apresentação da tecnologia em saúde, os profissionais mostraram-se satisfeitos e assertivos em aceitar a aplicação da Ficha de Avaliação de Feridas no ambiente assistencial, o qual em questão, trata- se da unidade de terapia intensiva. Assim, constata-se que uma atividade de Educação Permanente, pautada nas reais necessidades da população-alvo, pode provocar mudanças significativas, induzindo a promoção da saúde.

Em conseguinte, a Educação Permanente em saúde é adequada para desenvolver novas ideias no cenário das transformações para a execução do cuidado na Unidade de Terapia Intensiva. As estratégias de aprendizagem podem servir para desenvolver o pensamento crítico e dialógico, oportunizando um espaço de participação coletiva e contribuindo no processo de compreensão da realidade do trabalho e promoção de estratégias adequadas para a produção de novos conhecimentos em busca de transformações ${ }^{19}$.

À vista disso, a preparação e elaboração da atividade a partir de um problema identificado na experiência prática no campo de estágio supervisionado foram facilitados pela metodologia empregada, a partir da problematização.

\section{Considerações finais}

O presente estudo tencionou ônus à utilização de uma tecnologia em saúde, prática abrangente e objetiva que apresentou aceitação unânime, além de sensibilidade ao uso por parte da equipe, para aplicar ao paciente criticamente enfermo que necessita de contínua vigilância e suporte terapêutico especializado.

Considera-se, portanto, verossímil, a contribuição desta tecnologia para o engajamento do planejamento do cuidado ao paciente com a mobilidade comprometida, em grande risco de lesão por pressão, o que colabora na execução de ações rápidas e competentes para a prevenção de tais lesões, direcionando as intervenções de enfermagem, garantindo uma avaliação clínica minuciosa, além de gerar benefícios ao profissional, ao serviço e especialmente aos pacientes.

\section{Referências}

1. Balsanelli AP, Zanei SSV, Whitaker IY. Carga de trabalho de enfermagem e sua relação com a gravidade dos pacientes cirúrgicos em UTI. Acta Paul Enferm. 2006;19(1):16-20. http://dx.doi.org/10.1590/S0103-21002006000100003.

2. Geovanini T, Oliveira JAG. Manual de curativos. 2. ed. São Paulo: Corpos; 2010.

3. Fernandes LM. Úlceras de pressão em pacientes críticos hospitalizados. Uma revisão integrativa da literatura [dissertação]. Ribeirão Preto: Escola de Enfermagem de Ribeirão Preto, Universidade de São Paulo; 2000. http://dx.doi.org/10.11606/D.22.2000. tde-25112004-092213.

4. Esperón GJA, Castelo ACI, Escudero QA, et al. Monitorización de úlceras por presión en una Unidad de cuidados intensivos; 2007;18(3):40-48.

5. Echer IC, Santos JBP, Suzuki LMS, Antoniazzi JL. Avaliação e tratamento de feridas: orientações aos profissionais de saúde. Porto Alegre: Hospital de Clínicas de Porto Alegre; 2011. 
6. Fernandes LM. Efeitos de intervenções educativas no conhecimento e práticas de profissionais de enfermagem e na incidência de úlcera de pressão em centro de terapia intensiva [tese]. Ribeirão Preto: Escola de Enfermagem de Ribeirão Preto, Universidade de São Paulo; 2006.

7. Arone EM, Cunha I. Tecnologia e humanização: desafios gerenciados pelo enfermeiro em prol da integridade da assistência. Rev Bras Enferm. 2007;60(6):721-3. http://dx.doi.org/10.1590/S0034-71672007000600019. PMid:18472549.

8. Dantas NC, Pereira VE, Tourinho SVF. Nursing consultation as a technology for care in light of the troughts of Bacon and Galimberti. Texto Contexto Enferm. 2016;25(1):1-8.

9. Ramos BJ. Tecnologia do cuidado de enfermagem no manejo do paciente adulto com cateter venoso totalmente implantado:validação de um instrumento [dissertação]. Florianópolis: Universidade Federal de Santa Catarina; 2016.

10. Fernandes NC, Teixeira PRA, Sá AMM, Medeiros LM, Peixoto IVP. Monitoria acadêmica e o cuidado da pessoa com estomia: relato de experiência. Rev Min Enferm. 2015;19(2):238-41.

11. Xavier LN, Oliveira GL, Gomes AA, Machado MFAS, Eloia SMC. Analisando as metodologias ativas na formação dos profissionais de saúde: uma revisão integrativa. SANARE. 2014;13(1):76-83.

12. Mitre SM, Siqueira-Batista R, Girardi-de-Mendonça JM, et al. Metodologias ativas de ensino-aprendizagem na formação profissional em saúde: debates atuais. Cien Saude Colet. 2008;13(2, Supl 2):2133-44. http://dx.doi.org/10.1590/S141381232008000900018. PMid:19039397.

13. Berbel NAN. A problematização e a aprendizagem baseada em problemas: diferentes termos ou diferentes caminhos? Interface (Botucatu). 1998;2(2):139-54. http://dx.doi.org/10.1590/S1414-32831998000100008.

14. Brasil. Agência Nacional de Vigilância Sanitária - ANVISA. Assistência segura: uma reflexão teórica aplicada à prática [Internet]. Brasília: ANVISA; 2013 [citado em 2019 Mar 29]. Disponível em: https://www20.anvisa.gov.br/segurancadopaciente/ images/documentos/livros/Livro1-Assistencia_Segura.pdf

15. Brasil. Ministério da Saúde. Portaria n. 529, de $1^{\circ}$ de abril de 2013. Institui o Programa Nacional de Segurança do Paciente (PNSP). Brasília: Diário Oficial da União [Internet]. Brasília; 2013 [citado em 2019 Mar 29]. Disponível em: http://www. saude.mt.gov.br/upload/controle-infeccoes/pasta2/portaria-msgm-n-529-de-01-04-2013.pdf

16. Rocha KPWF. A educação em saúde no ambiente hospitalar. Rev Nursing. 2007;108(9):216-20.

17. Silva AC, Zanatta EA. Educação em saúde percepção de biólogos. Rev Nursing. 2009;131(12):177-81

18. Costa CR, Costa LM, Boução DMN. Escala de Braden: a importância da avaliação do risco de úlcera de pressão em pacientes em uma unidade de terapia intensiva. Revista Recien. 2016;6(17):36-44. http://dx.doi.org/10.24276/rrecien2358-3088.2016.6.17.36-44.

19. Paim CC, Ilha S, Backes SD. Educação permanente em saúde em unidade de terapia intensiva: percepção de enfermeiros. Rev Pesqui Cuid Fundam (Online). 2015;7(1):2001-2010.

\section{Contribuição dos autores}

Ana Zélia Silva Fernandes de Sousa contribuiu com a elaboração do projeto, desenvolvimento da atividade educativa e de todas as fases de elaboração do manuscrito. Joana Carla da Silva Souza participou na concepção inicial, análise e interpretação dos dados do manuscrito. Daniele Lima dos Anjos Reis, contribuiu com a elaboração do projeto, acompanhamento, desenvolvimento e orientação do estudo. Kátia Simone Kietzer contribuiu na orientação e análise do manuscrito. Tendo ambos participado da redação e revisão crítica do conteúdo. 